Psychopharmacology 48, 79-82(1976)

\title{
Effects of a Novel Anti-Aggressive Agent upon Two Types of Brain Stimulated Emotional Behavior*
}

\author{
R. J. KATZ and E. THOMAS \\ Mental Health Research Institute, University of Michigan, Ann Arbor, Michigan 48109, U.S.A. and \\ Department of Psychology, Bryn Mawr College, Bryn Mawr, Pennsylvania 19010, U.S.A.
}

Abstract. The effects of anti-agressive agent Sch 12679 were evaluated upon stable baselines of rage and predation elicited by electrical stimulation of the hypothalamus in cats. Sch 12679 depressed approach and terminal aspects of both forms of attack. This is consistent with previous reports, and suggests the drug is effective in reducing many forms of aggression including brain stimulated emotional behavior.

Key words: Aggression - Brain stimulation - Predation - Rage - Sch 12679.

Recent evidence from a number of sources suggests that Sch 12679, a novel psychoactive compound chemically unrelated to existing classes of drugs, may be effective in reducing many forms of aggressive behavior. Such evidence includes both clinical studies upon a number of human populations (i.e., Itil et al., 1972; Keskiner et al., 1971; Park et al., 1972) and extensive behavioral testing employing a variety of animal models of aggression (Barnett et al., 1974).

Brain stimulated emotional behavior offers highly specific, quantifiable, and stable measures of aggression (Flynn, 1967; Flynn et al., 1970). Such measures, in addition, are often not available or available only with great difficulty in other attack models. The advantages of brain stimulation in these regards have been witnessed in a number of recent studies of drug effects upon attack patterns and individual aspects of attack sequences [e.g., the recent studies of MacDonnell and his colleagues upon ethanol and related com-

* This research was supported by Grant MH 15946 from the
National Institute of Mental Health to the second author. We
are grateful to Schering Corporation for the generous donation are grateful
of $\operatorname{Sch} 12679$. pounds; MacDonnell and Ehmer, 1969; MacDonnell and Fessock, 1969; MacDonnell et al., 1971; MacDonnell, 1971, as well as the work of Katz and Thomas (1975) upon the aminergic control of predation]. Such factors render brain stimulated emotional behavior a useful baseline for further evaluating the anti-aggressive properties of Sch 12679.

In addition, the use of central stimulation in the cat extends considerably the behavioral domain in which Sch 12679 has been tested, allowing genralization to aggression elicited by the direct electrical activation of the central circuits underlying attack. The present studies were therefore designed to investigate the effect of Sch 12679 upon the approach and terminal components of the two major forms of aggression (i.e., rage and predation) induced by electrical stimulation of hypothalamic sites.

\section{EXPERIMENT 1}

\section{EFFECTS OF SCH 12679 \\ UPON BRAIN-STIMULATED RAGE}

\section{Methods}

Subjects. The subject pool for Experiment 1 consisted of 5 adult female cats weighing 2.0 to $3.5 \mathrm{~kg}$, obtained from a local supplier (Bio Medical Associates, Inc., Friedensburg, Pa.). All subjects were maintained with food and water continuously available throughout the experiment. Normal cycles of $14 \mathrm{~h}$ light and $10 \mathrm{~h}$ darkness were maintained by natural and artificial lighting.

Surgery. Subjects were anesthetized via intraperitoneal injection of $35 \mathrm{mg} / \mathrm{kg}$ sodium pentobarbital (Nembutal). Each subject was stereotaxically implanted with twelve stainless steel electrodes $0.25 \mathrm{~mm}$ in diameter insulated to the tip. In addition, two indifferent electrodes were attached to stainless steel screws for purposes of monopolar stimulation. All electrodes were aimed at the ventromedial hypothalamic nucleus. All plaements were based on the atlas of Snider and Neimer (1964). Surgery was performed under aseptic conditions. At the close of surgery 150000 units of Bicillin were administered intramuscularly. 
Apparatus. During testing for approach, subjects were maintained in an especially constructed apparatus that allowed continual testing of the subject with a minimum of interference from the experimenter. The aggression apparatus consisted of $50 \times 32 \times 111 \mathrm{~cm}$ wooden box with a central barrier $7.5 \mathrm{~cm}$ in height and a grid floor made of aluminium dowels. Apertures at either end of the apparatus allowed an attack object (a small stuffed toy $14 \mathrm{~cm}$ in length resembling a squirrel) to be maintained in constant irregular motion at the end of the box distal to the subject by means of one of two motor driven rods. A 2HBA-1 Unimax microswitch with a pressure requirement of $28 \mathrm{~g}$ and a release requirement of $21 \mathrm{~g}$ was mounted at the end of each rod. An attack object was attached to each microswitch by nylon fishing line.

To assess changes in approach two sets of photocells (Sigma models 8-L-3, 8-P-3) were mounted $14 \mathrm{~cm}$ above and $4 \mathrm{~cm}$ distal to the central barrier, along the width of the box. Doors of the apparatus consisted of one-way mirrors through which visual observations of attack could be made. A constant level of $20.0 \mathrm{DB}$ white noise was maintained throughout the experiment via a Grason-Stadler white noise generator.

To assess terminal aspects of attack the subject was immobilized in a canvas feline restraint bag (Lentz, Inc.) modified to allow free movement of either paw. Once the subject was immobilized, the attack object was brought into contact with the subject's mouth or paw. This procedure allowed approach free measures of terminal attack patterns, and assured that changes in approach would not be additionally reflected in changes in terminal measures. Procedure. One week after surgery animals were tested for responding to stimulation. Only sites that consistently yielded piloerection, growling, and directed attack were selected for additional testing. During initial testing and throughout the course of the experiments, stimulation consisted of monophasic pulses of $1.0 \mathrm{~ms}$ duration, delivered through a $2 \mathrm{uF}$ capacitor in series with the animal to allow reversal of current. Pulses were delivered 150 pulses $/ \mathrm{s}$ in 10-s trains, with intervals of 120 to $150 \mathrm{~s}$ between stimulations. All stimulation was delivered by a Grass SD-9 stimulator and monitored across a $100 \mathrm{ohm}$ resistor in series with the subject, on a 122-A Hewlett-Packard oscilloscope. Current levels were determined immediately prior to each stimulation by the presentation of a single $1.0 \mathrm{~ms}$ pulse.

Based upon initial protocols, subjects were tested for approach and terminal behaviors in the aggression apparatus. Two levels of current were employed: threshold (Thr) and suprathreshold (Sup). Threshold current was defined as the lowest level of stimulation for the elicitation of at least one attack episode from two consecutive stimulations (attack was defined as approach culminating in terminal object contact). In the case of terminal reflexes, threshold was defined as the lowest level of current for the elicitation of at least one such reflex during two consecutive stimulations. For all measures suprathreshold current was defined as a current value 0.05 to $0.1 \mathrm{ma}$ above threshold. Subjects were tested twice consecutively at each level of stimulation with order of stimulations randomized. Two current parameters were employed in order to maximize any drug effects that might be masked at one or the other level due to ceiling or floor effects. All testing for approach and reflex aspects of attack was carried out in a single session of 30 to $45 \mathrm{~min}$.

Two measures of approach were obtained for each stimulation. The first measure was the latency from stimulation onset to initial movement as measured by barrier crossing (initiation). The second measure was the latency from initial movement to object contact (locomotion). All latencies were timed electronically, using Lehigh Valley Electronics, Inc., 412-01 timers operated by the photocells and microswitches of the apparatus. If a subject failed to cross the central barrier, initiation and locomotion scores were recorded as $10 \mathrm{~s}$ each. If the barrier was crossed without object contact, the locomotion score was recorded as $10 \mathrm{~s}$.
Subjects were tested for reflexes of biting and pawing in the restraint bag already described. Threshold and suprathreshold levels of stimulation were employed to assess the effects of Sch 12679 upon each of these two terminal reflexes elicited by proximal stimulation of the lipline and anterior paw surfaces of the subject contralateral to the site of stimulation. Terminal reflexes were evaluated in terms of a probability of response measure based upon two stimulations at each level of current.

Two stages of testing were employed. These were: establishment of a set of baseline behaviors under the appropriate control injections and drug administration and subsequent testing of behaviors.

Drugs. Based upon earlier published reports (Barnett et al., 1974) and preliminary testing ${ }^{1}$ a dose of $10 \mathrm{mg} / \mathrm{kg}$ was found to be highly effective in reducing aggression. At this dosage all subjects appeared alert and free from gross locomotor disturbance. All dosages were administered in $10 \mathrm{mg} / \mathrm{ml}$ normal saline vehicle solution via intraperitoneal injection. All injections were administered 15 min prior to testing.

Histology. At the close of the experiment all cats were injected with an overdose of Nembutal, and perfused first with a solution of normal saline and subsequently with a solution of $10 \%$ formaldehyde in saline. Brains were removed, sliced in 40 micron sections, and examined using a modification of Skinner's photographic histologic technique (Skinner, 1971). All electrode placements that were used were in the ventromedial hypothalamic area.

Results. The effects of Sch 12679 upon brain-stimulated rage are presented in Figure 1 and 2 . It may be seen in Figure 1 that Sch 12679 increases latencies for each of 2 measures of approach behavior. The respective $t$ values for initiation and locomotion are 3.61 and 3.84 at threshold and 4.33 and 2.47 above threshold. All values are significant employing a two tailed $t$ test for paired observations with the exception of locomotion at suprathreshold stimulation; the latter is significant using a 1 tailed criterion. The use of a 1-tailed test seems justified given the experimental hypothesis.

The effects of Sch 12679 upon each of two terminal reflexes are presented in Figure 2. Significant reductions can be seen in biting both at and above threshold (the respective $t$ values are 6.53 and 3.50). In addition, the drug suppressed pawing at and above threshold. The reduction of pawing at threshold is significant using a 1-tailed test $(t=2.12)$, however, the suprathreshold difference fails to reach statistical significance $(t=1.37)$.

1 Preliminary testing consisted of the administration of dosages of 1,10 , and $25 \mathrm{mg} / \mathrm{kg}$ to 2 cats, with a subsequent evaluation of approach and reflexive behaviors using the method of Katz and Thomas (1975). The $1 \mathrm{mg} / \mathrm{kg}$ dose produced virtually no changes in either measure. The $10 \mathrm{mg} / \mathrm{kg}$ dose produced decrements ranging from $40-75 \%$ in each category, and the $25 \mathrm{mg} / \mathrm{kg}$ dose produced virtually total suppression of all behavior. The 2 cats were additionally tested upon $2 \times 5 \mathrm{~m}$ grid floor for locomotor activity. Activity was unchanged at all but the highest dose, at which point ataxia and incoordination were prominent. 


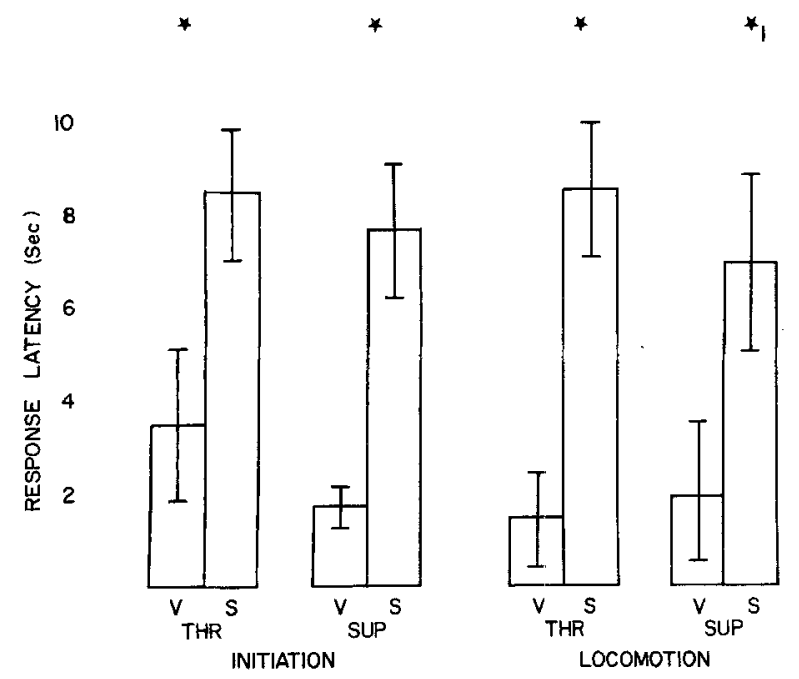

Fig. 1. Effects of Sch 12679 upon rage (approach behaviors). $\mathrm{V}=$ saline vehicles; $\mathrm{S}=\mathrm{Sch} 12679, \mathrm{Thr}=$ threshold current for behavioral elicitation; Sup = suprathreshold current for behavioral elicitation $10 \mathrm{mg} / \mathrm{kg},{ }^{*}=P<0.05,2$-tailed $t$ test for within subject differences; $* 1, P<0.05$, 1 -tailed criterion. All Figures are presented as means \pm standard errors of the mean
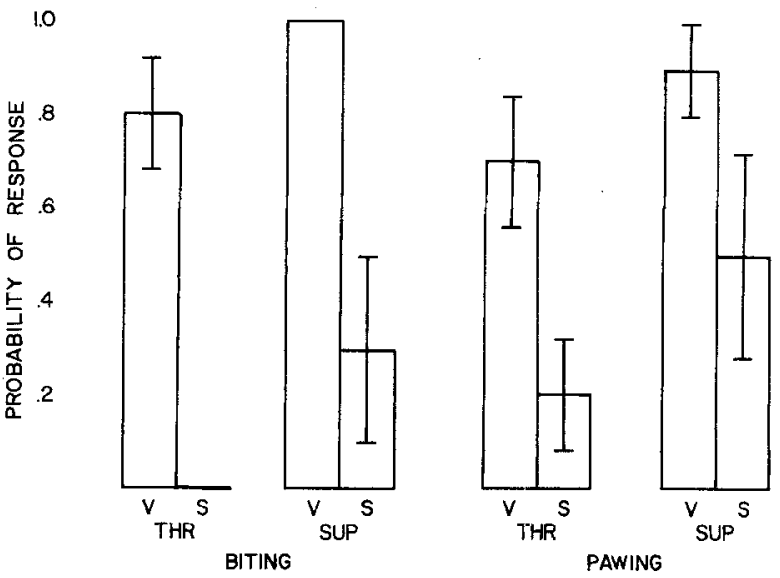

Fig.2. Effects of Sch 12679 upon rage (reflexive behaviors) V $=$ saline vehicle; $\mathrm{S}=\mathrm{Sch} 12679,10 \mathrm{mg} / \mathrm{kg}$, Thr = threshold current for behavioral elicitation; Sup = suprathreshold current for behavioral elicitation, ${ }^{*}=P<0.05$, 2-tailed $t$ test for within subjects differences; *1, $P<0.051$-tailed criterion. All Figures are presented as means \pm standard errors of the mean

\section{EXPERIMENT 2}

\section{EFFECTS OF SCH 12679 UPON CENTRALLY ELICITED PREDATORY ATTACK}

Method. Subjects were 5 adult female cats maintained under conditions identical to those previously reported in Experiment 1. Surgical procedures were identical to those previously reported, except that the electrode placements were aimed at lateral hypothalamic sites. Only sites that yielded quiet biting directed at the neck, and from which piloerection was absent, were selected for

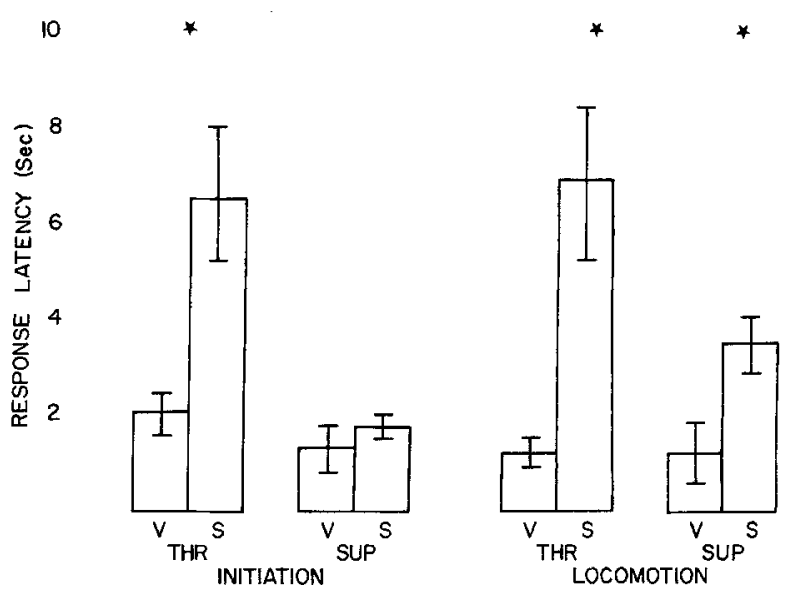

Fig. 3. Effects of Sch 12679 upon predation (approach behaviors). $\mathrm{V}=$ saline vehicle $; \mathrm{S}=$ Sch $12679,10 \mathrm{mg} / \mathrm{kg}$, Thr $=$ threshold current for behavioral elicitation; Sup $=$ suprathreshold current for behavioral elicitation, ${ }^{*}=P<0.052$-tailed $t$ test for within subjects differences. All Figures are presented as means \pm standard errors of the mean
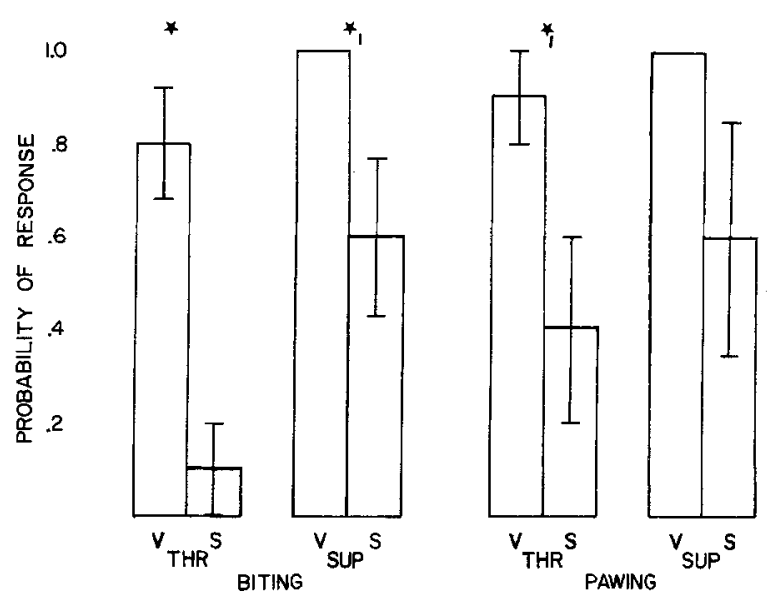

Fig. 4. Effects of Sch 12679 upon predation (reflexive behaviors). $\mathrm{V}=$ saline vehicles; $\mathrm{S}=\mathrm{Sch} 12679,10 \mathrm{mg} / \mathrm{kg}, \mathrm{Thr}=$ threshold current for behavioral elicitation; Sup $=$ suprathreshold current for behavioral elicitation, $*=P<0.052$-tailed $t$ test for within subject differences; $* 1=P<0.051$-tailed criterion. All Figures are presented as means \pm standard errors of the mean

drug testing. Apparatus, experimental, and histological procedures were identical to those previously reported All sites were located in the lateral hypothalamic and perifornical areas.

Results. Results are presented in Figure 3 and 4. Figure 3 presents drug effects upon initiation and locomotion. All approach latencies are prolonged. All differences are significant at threshold $(t$ scores are 2.96 and 3.65 , respectively for initiation and locomotion. In addition, locomotion is significantly depressed at suprathreshold levels of stimulation $(t=2.93)$, 
but the suprathreshold reduction in initiation fails to reach a normally accepted criterion of significance $(t=1.72)$.

Figure 4 presents the effects of the drug upon terminal reflexes. It may be seen that Sch 12679 reduces both reflexive biting and pawing. These reductions are significant for reflexive biting at threshold $(t=5.72)$, and significant for pawing at threshold, and biting above threshold, employing a 1-tailed criterion (values were 2.23 and 2.13 respectively). The reduction in suprathreshold patterns of pawing fails to reach statistical significance $(t=1.63)$.

\section{GENERAL DISCUSSION}

The present results are consistent with previously published findings regarding the anti-aggressive potential of Sch 12679, and serve to expand the latter to a novel species, models of attack, and measures of behavior.

It might be noted that in both studies the approach and terminal aspect of attack were affected by the drug manipulation. Similar results have been reported by Katz and Thomas (1975) subsequent to parenternal administration of anticholinergic agents, although such findings are not common to all drugs affecting aggression (e.g., AMPT; Katz and Thomas, 1975; also ethanol; MacDonnell and Ehmer, 1969). It might be argued from the common disruption of approach and terminal aspects of attack that Sch 12679 was effective because of a nonspecific depression of all activity. Although no formal measurements of non-aggressive activity were taken in the present study it was our overall impression that all cats ate, drank, and moved about normally upon being returned to their cages. In addition, we also have noted increases in salivation subsequent to Sch 12679 . The finding of increased salivation renders a general depression of all reflexes unlikely.

Finally, it might be noted the 3 reported clinical studies are in general agreement regarding the absence of sedation at those drug levels most therapeutic against aggression. These factors, while not eliminating the possibility for a sedative effect, render sedation inadequate as a comprehensive explanation for all the anti-aggressive effects of Sch 12679.

\section{REFERENCES}

Barnett, A., Taber, R. I., Steiner, S. S.: The benaviorai pticin. cology of Sch 12679: A new psychoactive agent. Psychopharmacologia (Berl.) 36, 281 - 290 (1974)

Dixon, W. J., Massey, F. J.: Introduction to statistical analysis. New York: McGraw-Hill 1969

Flynn, J. P.: The neutral basis of aggression in cats. In: Neurophysiology and emotion, D. C. Glass, ed. p. 40-60, New York: Rockefeller University 1967

Flynn, J. P., Vanegas, H., Foote, W., Edwards, S.: Neural mechanisms involved in a cat's attack on a rat. In: The neutral control of behavior, R. E. Whalen, R. F. Thompson, M. Verzeano, and N. M. Weinberger, eds., pp. 135-173. New York-London: Academic Press 1970

Itil, T. M., Stock, M. J., Duffy, A. D., Esquenazi, A., Saleuty, B., Han, T. H.: Therapeutic trials and EEG investigations with Sch 12679 in behaviorally disturbed adolescents. Curr. ther. Res. 14, 136-150 (1972)

Katz, R. J., Thomas, E.: Effects of scopolamine and $\alpha$-methylparatyrosine upon predatory attack in cats. Psychopharmacologia (Berl.) 42, 153-157 (1975)

Keskiner, A., Itil, T. M., Han, T. H., Saleuty, B., Hsu, W.: Clinical toxicological, and electroencephalographic study with Sch 12679 in chronic scizophrenics. Curr. ther. Res. 13, 714- 725 (1971)

MacDonnell, M. F., Ehmer, M.: Some effects of ethanol on agressive behavior in cats. Quart. J. Stud. Alcohol 30, 312 - 319 (1969)

MacDonnell, M. F., Fessock, L. : Disulfiram and brain-stimulated emotional behavior. Quart. J. Stud. Alcohol 30, 719-723 (1969)

MacDonnell, M. F., Fessock, L., Brown, S. H.: Ethanol and the neural substrate for affective defense in the cat. Quart. J. Stud. Alcohol 32, 406-419 (1971 a)

MacDonnell, M. F.: Aggression and associated neural events in cats; effects of p-chlorophenylalanine compared with alcohol. Quart. J. Stud. Alcohol 32, 748-763 (1971 b)

Park, S., Gershon, S., Floyd, A.: A clinical trial of benzazepine (Sch 12679) in acute schizophrenic patients. Curr. ther. Res. 14, 298-302 (1972)

Skinner, J. E.: Neuroscience: a laboratory manual. Philadelphia: Saunders 1971

Received September 26, 1975; Final Version January 15, 1976 